\title{
Method to measure the localization length in one dimension
}

\author{
Zhao-Qing Zhang and King-Chuen Chiu \\ Department of Physics, Hong Kong University of Science and Technology, Clear Water Bay, Kowloon, Hong Kong \\ Daozhong Zhang \\ Institute of Physics, Chinese Academy of Sciences, Beijing, China
}

(Received 12 July 1996)

\begin{abstract}
We propose a method to measure the localization length in one dimension. By relating the width of a resonant mode to the diffusion time, the localization length of the resonant mode can be measured. By taking the average in frequency space, we demonstrate explicitly that the localization length of an infinite sample can be reproduced accurately from a finite sample of size comparable to, or even smaller than, the localization length itself. Experimental support for this approach is presented. [S0163-1829(96)07042-7]
\end{abstract}

During the past two decades, much attention has been focused on the transport and localization behaviors of electrons and classical waves in disordered materials. ${ }^{1,2}$ Despite the tremendous progress that has been made in this area, direct experimental measurement of a key quantitylocalization length - is still difficult if not rare. In the case of electrons, the localization of electrons is often complicated by the presence of inelastic scattering and electron-electron interaction effects. ${ }^{1}$ In the case of classical waves, the standard way of measuring the localization length is to obtain the transmission coefficient $T$, at any given frequency $\omega$, as a function of sample size $L$, much larger than the localization length $\xi(\omega)$, i.e., $T \approx \exp (-L / \xi)$. In this localized regime, the self-averaging effect provides a reliable measurement of the localization length at that frequency. Theoretically, this can be done by using the transfer-matrix approach. ${ }^{3,4}$ Experimentally, this is a difficult task because the transmission coefficient decays exponentially with sample size, making accurate measurement impossible. Very often, the presence of absorption in the medium adds another decay length to the system so as to mask the localization characteristics. However, if the sample size is comparable to the localization length, the widths of resonant modes in the transmission spectrum become comparable to the level spacing. ${ }^{5,6}$ At each resonant mode $\omega_{r}, T$ is on the order of 1 . The value of $T$ reduces according to a Lorentzian form as $\omega$ moves away from $\omega_{r}$. Thus, the localization length obtained from the relation $T \approx \exp (-L / \xi)$ contains strong sample and frequency fluctuations. Averaging of many samples is required.

In this paper, we propose an approach to measure the localization length in one dimension in the regime where sample size is comparable to the localization length. We utilize the information provided by the width of a resonant mode in frequency space and relate them to the diffusion time. The inverse of the width measures the time it takes for the waves to tunnel through the sample. This dwell time is equal to the diffusion time for the wave to travel across the sample, apart from a numerical factor. Since in one dimension the localization length is on the order of transport mean free path, the localization length, $\bar{\xi}\left(\omega_{r}\right)$, at each resonant mode, $\omega_{r}$, can, therefore, be measured from the width of the resonant mode in the transmission spectrum. The value of $\bar{\xi}\left(\omega_{r}\right)$ fluctuates from mode to mode. If there exists many resonant modes in a frequency window $(\omega+\delta \omega, \omega-\delta \omega)$ where localization length of an infinite sample, $\xi(\omega)$, varies slowly, we are able to reproduce accurately the value of the $\xi(\omega)$ by taking the average in this frequency window, i.e., $\left\langle\bar{\xi}\left(\omega_{r}\right)\right\rangle$. By using this method, we show explicitly in 1D that the localization length of electromagnetic waves can be accurately obtained from a single sample of size comparable to, or even smaller than, the localization length itself. Both homogeneously random systems and disordered systems that are periodic on average ${ }^{4,7}$ have been studied. We have also applied this method to the existing experimental transmission spectra. ${ }^{8}$ The results are in good agreement with those of numerical simulations.

Consider a disordered layered sample of $L$ layers, where both the dielectric constant and layer thickness are random. The dielectric constant in the $n$th layer has the value $\varepsilon_{n}=\varepsilon_{0}\left(1+\sigma \eta_{n}\right)$, where $\varepsilon_{0}$ is the effective dielectric constant, $0<\sigma<1$ gives the amplitude of randomness, and $\eta_{n}$ is a random number between $(-1,1)$. The thickness $\Delta z$ of a given layer is assumed to follow an exponential distribution $b^{-1} \exp (-\Delta z / b)$, where $b$ is the mean layer thickness. Here we set $b=1$. The sample is embedded in a homogeneous medium with wave speed $c=c_{0} / \sqrt{\varepsilon_{0}}$, where $c_{0}$ is the wave speed in the vacuum. For a nonmagnetic medium, the timeindependent Maxwell equation for the electric field of a normally incident wave is ${ }^{9}$

$$
\frac{\partial^{2} E(z)}{\partial z^{2}}+\frac{\omega^{2}}{c_{0}^{2}} \varepsilon(z) E(z)=0,
$$

where $\omega$ is the frequency of the wave. The transmission coefficient $T$ can be calculated by using the transfer matrix. The localization length behavior of such a system has been studied before. ${ }^{3,9}$ In the long-wavelength limit $(\lambda=2 \pi c /$ $\omega \gg 1)$, the localization length $\xi$ goes quadratically with $\lambda$, i.e., $\xi \cong 12(c / \sigma \omega)^{2}$. In the short-wavelength limit $(\lambda<2), \xi$ saturates to a constant value, which depends on $\sigma$.

If we add a constant negative imaginary part to the dielectric constant in every layer, i.e., $\varepsilon_{n}=\varepsilon_{0}\left(1+\sigma \eta_{n}\right)-i \varepsilon^{\prime \prime}$, the light becomes amplifying while traveling in the sample. This amplification adds a gain length $l_{g}$ to the system. In a homo- 
geneous medium, this length is the inverse of the imaginary part of the wave vector, i.e., $l_{g}=2 c \varepsilon_{0} / \omega \varepsilon^{\prime \prime} .{ }^{9}$ In a random medium, the localization of waves produces many resonant cavities. At each resonant frequency $\omega_{r}$ there exists a threshold amplification $\varepsilon_{r}^{\prime \prime}$ at which $T$ diverges. This divergence occurs at discrete number of poles in the transmission matrix $t$ in the $\omega-\varepsilon^{\prime \prime}$ plane, where $T=|t|^{2}$. A statistical relation that gives the onset of the divergence of $\langle T\rangle$ has been found recently, ${ }^{9}$ i.e.,

$$
\xi \cong \frac{\omega \varepsilon^{\prime \prime} L^{2}}{2 c \varepsilon_{0}} \quad \text { or } \quad \xi l_{g} \cong L^{2}
$$

Equation (2) is valid only when the renormalized length $\Lambda \equiv L / \xi$ falls in the range of $0.3<\Lambda<3$. At resonant modes, Eq. (2) provides a relation between the threshold gain and the localization length of a particular mode, i.e., $\bar{\xi}\left(\omega_{r}\right)$ $\cong \omega_{r} \varepsilon_{r}^{\prime \prime} L^{2} /\left(2 c \varepsilon_{0}\right)$. The physical interpretation of this relation is the following. In one dimension, the localization length $\xi$ is about two to four times the transport mean free path $l .{ }^{9,10}$ Therefore, at the threshold value of $\varepsilon^{\prime \prime}$ of a resonant mode, the path length of the light traveling across a sample of thickness $L$ becomes comparable to the gain length, i.e., $L^{2} / l \cong l_{g}$. Here, the difficult part is to know the value of $\varepsilon_{r}^{\prime \prime}$ at each mode $\omega_{r}$. We show below that $\varepsilon_{r}^{\prime \prime}$ is directly related to the width of the resonant peak at $\omega_{r}$ measured from the transmission spectrum of a nonamplifying medium.

In the case of $\varepsilon^{\prime \prime}=0$, the transmission spectrum $T(\omega)$ contains many resonant peaks with a Lorentzian form

$$
T(\omega)=\frac{A\left(\omega_{r}\right)}{\left(\omega-\omega_{r}\right)^{2}+\Delta \omega_{r}^{2}},
$$

where $\Delta \omega_{r}$ and $A\left(\omega_{r}\right) / \Delta \omega_{r}^{2}$ are, respectively, the half-width and height of the peak at $\omega_{r}$. Equation (3) represents a pole in the transmission matrix $t$ at $\omega_{r}-i \Delta \omega_{r}$ in the complex $\omega$ plane. However, this pole can only be reached if the medium has gain, i.e., $\varepsilon^{\prime \prime}>0$. To the lowest order approximation, the presence of $\varepsilon^{\prime \prime}$ adds a term $-i c / l_{g}$ to the imaginary part of $\omega$. Therefore, at $\omega_{r}$, the complex frequency becomes $\omega_{r}-i \omega_{r} \varepsilon^{\prime \prime} /\left(2 \varepsilon_{0}\right)$. Thus, we would expect the following relation to hold at the threshold value of $\varepsilon^{\prime \prime}$ :

$$
\varepsilon_{r}^{\prime \prime} \cong 2 \varepsilon_{0} \Delta \omega_{r} / \omega_{r} .
$$

By using this relation and Eq. (2), we have

$$
\bar{\xi}\left(\omega_{r}\right) \cong a \frac{\Delta \omega_{r} L^{2}}{c},
$$

where $a$ is a constant on the order of 1 and will be determined numerically. Apart from this numerical factor, Eq. (5) states that the dwell time, $1 / \Delta \omega$, the light staying inside the sample is equal to the diffusion time. To verify Eqs. (4) and (5), we have set $\varepsilon_{0}=c=1$ in our numerical simulations for the case of homogeneously random systems. At each given value of $\sigma=0.3,0.5$, and 0.9 , we have chosen three sample thicknesses $L=n \xi_{s}$, with $n=1,2$, and 3, where $\xi_{s}$ is the saturated localization length in the large $\omega$ limit and has values about 510,170 , and 34 for $\sigma=0.3,0.5$, and 0.9 , respectively. Thus, the renormalized sample thickness becomes $\Lambda=1,2$, and 3 in the saturation regime. For a ran-

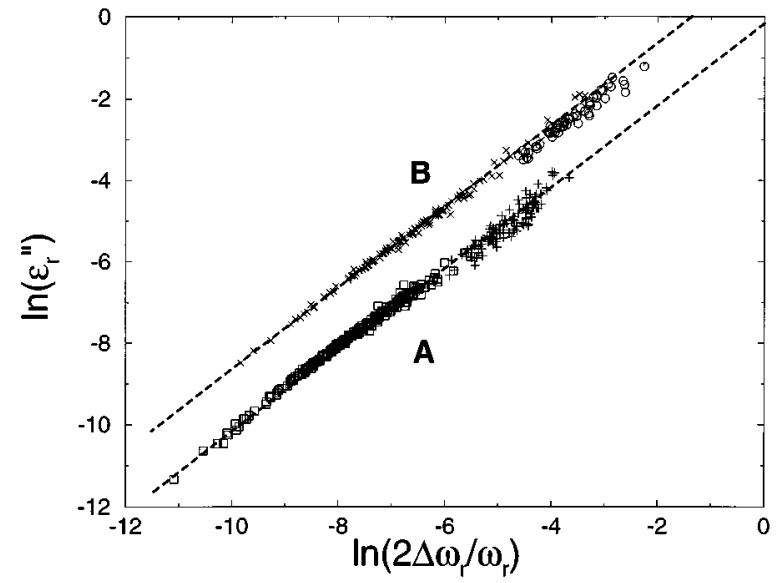

FIG. 1. The $\log -\log$ plot of $2 \Delta \omega_{r} / \omega_{r}$ vs $\varepsilon_{r}^{\prime \prime}$ at resonant modes. The "squares" and "pluses" in branch $A$ are the data of homogeneously random systems with $\sigma=0.5, \Lambda=2$ and $\sigma=0.9, \Lambda=1$, respectively. The "crosses" and "circles" in branch $B$ are the data of random binary system at wavelengths outside and inside the bandgap, respectively. The dashed curves represent Eq. (6).

domly generated configuration from a chosen set of $\sigma$ and $\Lambda$, we first calculate the transmission spectrum in the absence of gain by using the transfer matrix for $\omega$ in the range of $2 \pi / 25<\omega<2 \pi \quad(1<\lambda<25)$, which covers both shortwavelength and long-wavelength regimes. Then we select all the peaks that have well-defined Lorentzian shape. At each $\omega_{r}$, we measure the half-width $\Delta \omega_{r}$ and recalculate the transmission coefficient $T$ by adding the gain to the medium. The threshold gain $\varepsilon_{r}^{\prime \prime}$ is determined by the value of $\varepsilon^{\prime \prime}$ where $T$ has a maximum. We mark all pairs of $\varepsilon_{r}^{\prime \prime}$, and $2 \Delta \omega_{r} / \omega_{r}$ by points in a plane with $\ln \left(2 \Delta \omega_{r} / \omega_{r}\right)$ as the $x$ axis and $\ln \left(\varepsilon_{r}^{\prime \prime}\right)$ as the $y$ axis. In Fig. 1 (branch $A$ ), we show these points by the symbols "square" and "plus" for the case of $\sigma=0.5, \Lambda=2$ and $\sigma=0.9, \Lambda=1$, respectively. All these points follow a straight dashed line with slope 1 . This is true for all other values of $\sigma$ and $\Lambda$ considered here.

By substituting the value of $\Delta \omega_{r}$ at each mode into Eq. (5) and choosing the value of $a$ to be 1.5 , we obtain a set of $\bar{\xi}\left(\omega_{r}\right)$. This set is represented by small "circles" in Fig. 2 for the case of $\sigma=0.3$ and $\Lambda=2$. Each circle represents an eigenmode of the sample and the corresponding localization length. The values of $\bar{\xi}\left(\omega_{r}\right)$ seem to fluctuate randomly from mode to mode. We then take an average over a window where the localization length does not change much. The window sizes we have chosen are 0.2 for $0.6<\omega<2 \pi$ and 0.03 for $2 \pi / 25<\omega<0.6$. The averaged result is represented by heavy and open "triangles" in the above two regions, respectively. Comparing this result with $\xi(\omega)$ calculated by the transfer matrix (the dashed curve), we find excellent agreement between the two for the entire range of frequencies considered. It is worth noting that the largest localization length obtained here is $\xi(0.29)=2167$. This number is about twice the sample thickness $(L=1020)$. The reason that we can determine a localization length which is larger than the sample size is due to the validity of Eq. (2) in the range where $0.3 \xi<L<3 \xi$. We have repeated the calculations for other values of $\sigma$ and $\Lambda$. These results are shown in branches $A, B$, and $C$ of Fig. 3 for $\sigma=0.3,0.5$, and 0.9 , respectively. 


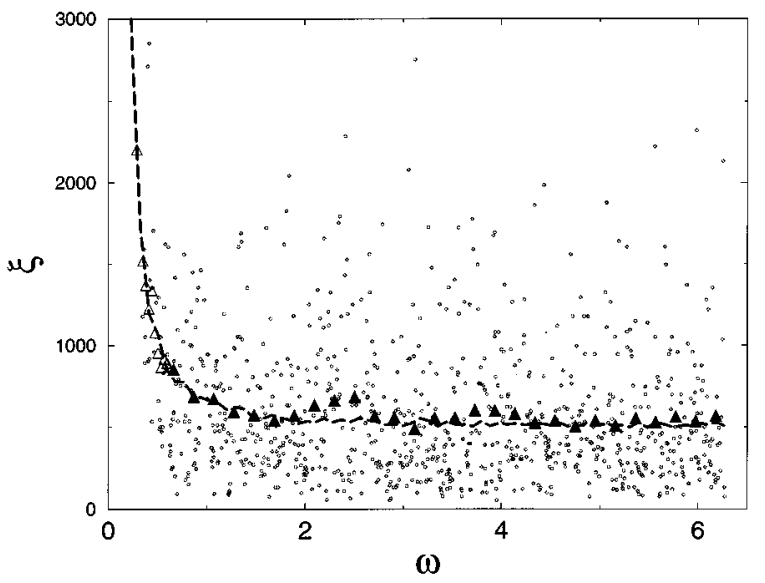

FIG. 2. The localization lengths calculated from Eq. (7) at various resonant modes are represented by small circles for the case of $\sigma=0.3$ and $\Lambda=2$. The open and solid triangles are the results of window average in different regions of frequency.

In each branch, the data obtained from single configuration with $\Lambda=1,2$, and 3 are represented by the symbols " $\times$," " $\triangle$," and " $\bigcirc$, ," respectively. The values of $a$ used in Eq. (5) are in the range of $1.45 \leqslant a \leqslant 1.55$. From the good agreement between these results and the dashed curves obtained from the transfer matrix, we can conclude that this method can apply to a wide range of randomness and frequency. Now we apply this method to a disorder system that is periodic on average.

A random binary system consists of alternating layers of $A$ and $B$ types. In order to test this method against the available experimental data, ${ }^{8}$ we have chosen the following specific system to study. Type $A$ consists of cryolite layers of equal thickness $d_{a}=122 \mathrm{~nm}$. Type $B$ consists of antimonite layers of random thickness $d_{b}=(57+W \eta / 2) \mathrm{nm}$, where $W$ describes the strength of disorder and $\eta$ is a random number between $(-1,1)$. The indices of refraction are $n_{a}=1.33$ $\left(\varepsilon_{a}=1.77\right)$ and $n_{b}=2.83\left(\varepsilon_{b}=8.00\right)$. The average thickness of a period is $L_{p}=179 \mathrm{~nm}$. When $W=0$, the dispersion relation

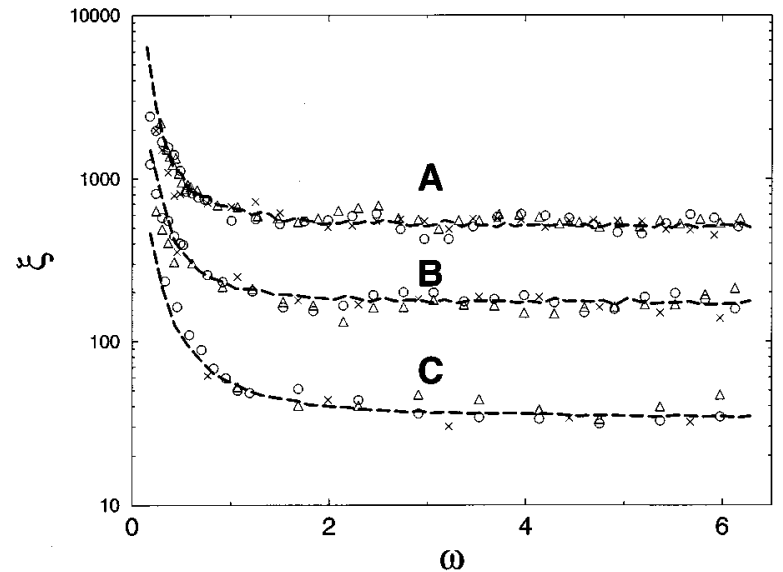

FIG. 3. The averaged localization lengths obtained from Eq. (7) are plotted in branch $A, B$, and $C$ for $\sigma=0.3,0.5$, and 0.9 , respectively. The crosses, triangles, and circles are the data obtained from $\Lambda=1,2$, and 3, respectively. The dashed curves are the results of transfer-matrix calculations.

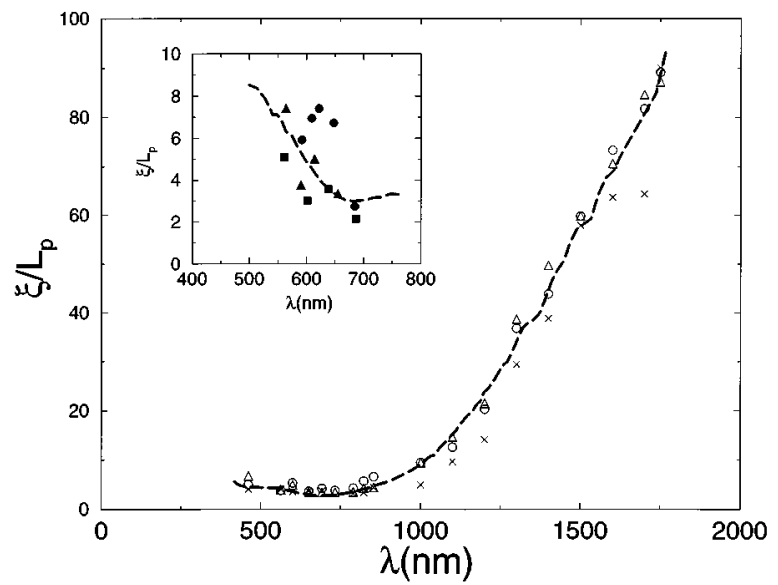

FIG. 4. The averaged localization lengths obtained from Eq. (7) are plotted in the neighborhood of the first bandgap. The crosses, triangles, and circles are the data obtained from $\Lambda=1,2$, and 3 , respectively. The inset shows the data obtained from experimental transmission spectra inside the bandgap for samples of 10 (squares), 15 (triangles), and 21 (circles) periods. The dashed curves are the results of transfer-matrix calculations.

shows that the first band gap appears in the region $529<\lambda$ $<849 \mathrm{~nm}$. We are interested in the localization length in the neighborhood of this band gap, i.e., $450<\lambda<1800 \mathrm{~nm}$. The value of $W$ used is 100 , which gives a standard deviation of $0.16 L_{p}{ }^{8}$ The localization length calculated by the transfer matrix is shown by a dashed curve in Fig. 4. Here we have used $L_{p}$ as the unit of localization length. Outside the band gap, we use nine windows. In each window $n$, three different $L$ 's are chosen according to $\Lambda=L / \xi\left(\lambda_{n}\right)=1,2$, and 3. In order to obtain better statistics of resonant modes, 10-20 configurations have been generated. Inside the band gap, we use a smaller window size due to relatively large change in $\xi$. Ninety configurations are generated. The correlations between $\varepsilon_{r}^{\prime \prime}$ and $2 \Delta \omega_{r} / \omega_{r}$ are shown by points in branch $B$ of Fig. 1. The symbols " $X$ " and " $O$ " represent the data obtained from outside and inside the band gap, respectively. They all follow a straight dashed line in branch $B$, which is parallel to the dashed line in branch $A$. The distance between the two lines arises from the difference in the effective dielectric constant $\varepsilon_{0}$ in Eq. (4). From the value of $\varepsilon_{0}(\cong 4.48)$ deduced from Fig. 1, we find an effective medium speed $c \cong 0.47 c_{0}$. This value represents a mean phase velocity in the region we considered, where the phase velocity at $\lambda=1800 \mathrm{~nm}$ is $0.51 c_{0}$ and reduces to $0.43 c_{0}$ at the band edge $(\lambda=849 \mathrm{~nm})$. Thus, Fig. 1 shows that Eq. (6) is valid in general if a proper value of $\varepsilon_{0}$ (or $c$ ) is used. Using $c=0.47 c_{0}$ and $a=1.5$ in Eq. (5), we calculate the localization length at each resonant frequency. The averaged data are plotted in Fig. 4, where the symbols " $\times$," " $\triangle$," and " $\bigcirc$ ", represent the results obtained from $\Lambda=1,2$, and 3 , respectively. Again, our results are in good agreement with that of the transfer matrix.

In the following, we show the experimental support for this method. The transmission spectra from three samples of thickness $L / L_{p}=10,15$, and 21 have been measured experimentally in the wavelength region of interest here. ${ }^{8} \mathrm{~A}$ total of 13 resonant modes are found inside the gap. Due to the pres- 
ence of absorption in the antimonite films when $\lambda<650 \mathrm{~nm}$, the part of the measured width of a resonant peak arising from absorption should be subtracted from the total width. By taking this into account, Eq. (5), in terms of wavelength, now becomes

$$
\bar{\xi}\left(\lambda_{r}\right) \cong 1.5 \frac{2 \pi c_{0} L^{2}}{\lambda_{r}}\left(\frac{\Delta \lambda_{r}}{c \lambda_{r}}-\frac{x n^{\prime \prime}}{c_{0}}\right),
$$

where $x$ represents the volume fraction of the antimonite layers, which has the value 0.32 , and $n^{\prime \prime}$ is the imaginary part of the index of refraction of the antimonite layers. The values of $n^{\prime \prime}$ we used are obtained from the interpolation of the measured values of 65-nm thickness antimonite film, which are $n^{\prime \prime}=0,0.03$, and 0.06 for $\lambda=640,578$, and $546 \mathrm{~nm}$, respectively. ${ }^{11}$ Using Eq. (6), the localization lengths at resonant modes, $\bar{\xi}\left(\lambda_{r}\right)$, are plotted in the inset of Fig. 4, where the solid squares, triangles, and circles are the data obtained from 10, 15, and 21 periods samples, respectively. The dashed curve in the inset is the result of transfer-matrix calculations using random arrangements of 20 different antimonite layer thicknesses appearing in the samples. The predicted localization lengths seem to agree reasonably well with the calculated result.

In conclusion, we have proposed a way to measure the localization length of a finite sample in the regime where sample thickness $L$ is comparable to the localization length $\xi$. By measuring the width of a resonant mode, the corresponding localization length $\bar{\xi}\left(\omega_{r}\right)$ can be obtained. The density of these modes is proportional to the sample thickness. Thus, when $L$ is large, the localization length of an infinite sample can be accurately reproduced by taking the average of $\bar{\xi}\left(\omega_{r}\right)$ in a frequency window. It is worth pointing out that the regime of $L \approx \xi$ represents a transition regime between ballistic and localized regimes in $1 \mathrm{D}$. There does not exist a diffusive regime as $\xi \approx 2-4 l .^{9,10}$ Nonetheless, the diffusion character seems to manifest itself through the width of a resonant mode as it has been demonstrated here. As the localized regime is approached when $L \gg \xi$, our numerical simulations have indicated that Eq. (2) follows the behavior $\exp (-L / \xi) \propto \xi / l_{g}$. This implies that Eq. (5) should be replaced by $\exp \left[-L / \bar{\xi}\left(\omega_{r}\right)\right] / \bar{\xi}\left(\omega_{r}\right) \propto \Delta \omega_{r} / c$. This exponential behavior of the width in the localized regime is not unexpected. Finally, we expect that this method is also applicable to $2 \mathrm{D}$ and $3 \mathrm{D}$ systems in the strong scattering limit, where the localization length $\xi$ is on the order of transport mean free path $l$ as Eqs. (2)-(5) do not depend on the dimensionality of the system.

The authors thank P. Sheng and C. T. Chan for useful discussions. The work was supported in part by Hong Kong Research Grant Council (RGC) under Contract No. HKUST 617/95P.
${ }^{1}$ P. A. Lee, Rev. Mod. Phys. 57, 287 (1985).

${ }^{2}$ Scattering and Localization of Classical Waves in Random Media, edited by P. Sheng (World Scientific, Singapore, 1990).

${ }^{3}$ P. Sheng, B. White, Z. Q. Zhang, and G. Papanicolaou, Phys. Rev. B 34, 4757 (1986).

${ }^{4}$ A. R. McGurn, K. T. Christensen, F. M. Mueller, and A. A. Maradudin, Phys. Rev. B 47, 13120 (1993).

${ }^{5}$ M. Ya. Azbel, Phys. Rev. B 28, 4106 (1983).

${ }^{6}$ A. Z. Genack, in Scattering and Localization of Classical Waves in Random Media (Ref. 2), p. 207.

${ }^{7}$ K. M. Ho, C. T. Chan, and C. M. Soukoulis, Phys. Rev. Lett. 65 , 3152 (1990); S. John, Phys. Today 44 (5), 32 (1991).

${ }^{8}$ D. Z. Zhang, W. Hu, Y. L. Zhang, Z. L. Li, B. Y. Cheng, and G. Z. Yang, Phys. Rev. B 50, 9810 (1994).

${ }^{9}$ Z. Q. Zhang, Phys. Rev. B 52, 7960 (1995).

${ }^{10}$ E. N. Economou, C. M. Soukoulis, and A. D. Zdetsis, Phys. Rev. B 30, 1686 (1984).

${ }^{11}$ H. Schopper, Z. Phys. 131, 217 (1952). 